Pleione 12(1): 132 - 142. 2018.

ISSN: 0973-9467

(C) East Himalayan Society for Spermatophyte Taxonomy

doi:10.26679/Pleione.12.1.2018.132-142

\title{
Additions to the flora of Manipur state, North-Eastern India
}

\author{
N. Dhatchanamoorthy ${ }^{1,3}$, K. Ravikumar ${ }^{1}$ and Kreni Lokho ${ }^{2}$ \\ ${ }^{1}$ National Herbarium of Medicinal Resources used in Indian Systems of Medicines, \\ Centre for Conservation of Medicinal Resources, \\ The University of Trans-Disciplinary Health Sciences and Technology, \\ Foundation for Revitalisation of Local Health Traditions (FRLHT), \\ 74/2, Jarakabande Kaval, Attur Post, Via - Yelahanka, Bangalore - 560 064, India \\ ${ }^{2}$ Department of Botany, Madras Christian College, Tambaram, Chennai - 600 059, India \\ ${ }^{3}$ Author for correspondence: E-mail: dhatcha@gmail.com
}

[Received 07.06.2018; Revised 27.06.2018; Accepted 28.06.2018; Published 30.06.2018]

\begin{abstract}
Botanical surveys conducted at many places in Manipur state of India resulted in addition of 11 angiosperm taxa [Celtis tetrandra Roxb. (Cannabaceae), Crotalaria assamica Benth. (Leguminosae), Leucas lavandulifolia (Lamiaceae), Ilex excelsa (Wall.) Voigt (Aquifoliaceae), Illigera khasiana C.B.Clarke (Hernandiaceae), Jasminum dispermum Wall. (Oleaceae), Maesa chisia Buch.-Ham. ex D.Don (Primulaceae), Mycetia radiciflora (C.B. Clarke) Airy Shaw (Rubiaceae), Rotheca farinosa (Roxb.) Govaerts (Lamiaceae), Symplocos lucida (Thunb.) Siebold \& Zucc. (Symplocaceae) and Trichodesma khasianum C.B. Clarke (Boraginaceae)] to the stateflora. The information is presented here with updated nomenclature, brief botanical description, phenology, habitat, distribution, specimens examined and photographic evidence.
\end{abstract}

Key words: Angiosperm, Flora, Manipur, New additions, North-East India

\section{INTRODUCTION}

Manipur is one of the eight North-Eastern states of India. It is bordering on the north by Nagaland, on the East by Myanmar, partly on the south by Mizoram and Chin hills of Myanmar, and on the west by Cachar district of Assam. Survey and collection of medicinal plants across the country is the mandate for the Center of Excellence program supported by the Ministry of Environment and Forests \& Climate Change (MoEF\&CC), assigned to Foundation for Revitalisation of Local Health Traditions (FRLHT), Bangalore. Under this program, botanical surveys were undertaken at different places in Manipur state namely, Agiidziikhe, Asufii, Chokhurii, Esii Phi, Kapengho, Kapenho Karale, Karong, Khole, Khungho, Kozii, Koziir Korii, Makhan Khulloen, Punanamai, Sholitokhu, Soprie and Vanee during the years 2011 and 2017. These botanical surveys have resulted in the collection of 267 plant taxa. These plants were identified and confirmed by referring floras, revisions, monographs, research articles and recent publications (Hooker 1872-1897; Kanjilal et al., 1934-1940; Balakrishnan, 1981 \& 1983; Deb, 1981 \& 1983; Chowdhery \& Wadhwa, 1984; Haridasan \& Rao, 1985 \& 1987; Naithani, 1990; Sanjappa, 1992; Chaudhuri, 1993; Sharma and Sanjappa, 1993; Hajra et al., 1995a \& 1995b; Chauhan, 1996; Chowdhery et al., 1996 \& 2008; Ansari, 2008; Oleg Polunin \& Adam Stainton,1997; Singh et al., 2000a \& 2000b; Singh, 2001; Kumar \& Singh, 2001; Singh et al., 2002; Kazhuhrii Eshuo \& Chaturvedi 2011; Balakrishnan et al., 2012; Moaakum \& Dey, 2013; Thongam et al., 2013a \& 2013b; Balachandran \& Ravikumar, 
2013; Moaakum et al., 2014; Devi et al., 2018). Further critical studies revealed that 11 species viz., Celtis tetrandra (Ulmaceae), Crotalaria assamica (Leguminosae), Leucas lavandulifolia (Lamiaceae), Ilex excelsa (Aquifoliaceae), Illigera khasiana (Hernandiaceae), Jasminum dispermum (Oleaceae), Maesa chisia (Myrsinaceae), Mycetia radiciflora (Rubiaceae), Rotheca farinosa (Lamiaceae), Symplocos lucida (Symplocaceae) and Trichodesma khasianum (Boraginaceae) have been confirmed as new additions to the flora of Manipur. All the plant taxa were presented here in alphabetical order with updated nomenclature, family, phenology, habitat, distribution and collection details. All the voucher specimens are deposited at FRLH National Herbarium, Bangalore.

\section{Taxonomic Enumerations}

Celtis tetrandra Roxb., Fl. Ind. 2: 63. 1832; Hook.f., Fl. Brit. Ind. 5: 482. 1888; Kanjial et al., Fl. Assam 4: 277. 1940; A.S. Chauhan in Hajra, Contrib. Fl. Namdapha 282. 1996. C. trinervia Roxb., Fl. Ind. 2: 65. 1832. C. seretina Planch. in Ann. Sci. Nat. ser. 3, 10: 300. 1848; Wight, Ic. Pl. Ind. Or. 6:t. 1970. 1833. [Cannabaceae; Plate I a]

Trees, up to $8 \mathrm{~m}$ tall; branchlets lenticellate, wrinkled horizontally, glabrous. Leaves simple, alternate, $5-14 \times 2.5-7 \mathrm{~cm}$, obliquely ovate or obliquely ovate-lanceolate, asymmetric at base, margin serrate at upper half, entire at lower half, acuminate at apex, membranous, glabrous above, puberulous beneath; 3-nerved from base, prominent; lateral nerves 1-4 pairs, pinnate, prominent; intercostae scalariform, prominent. Stipules linear-lanceolate, lateral, free; petiole 3-10 mm long, slender, pubescent, shallowly grooved above. Fruiting peduncle thick, c. $8 \mathrm{~mm}$ long, glabrous. Drupes globose, c. $8 \mathrm{~mm}$ across, orange-red when ripe, glabrous.

Fruiting: September - January.

Habitat: Rare in evergreen forest; growing with Daphne papyracea Wall. ex G.Don, Galinsoga parviflora Cav., Oxyspora paniculata (D.Don) DC., Rhododendron sp. and Rubus moluccanus L.

Distribution: Bangladesh, Bhutan, Indonesia, Myanmar, Nepal, Thailand, Vietnam and India. In India, it has been reported from Arunachal Pradesh, Assam and now from Manipur.

Specimens examined: INDIA: Manipur, Imphal District, way to Noney from Imphal, 1450 $\mathrm{m}, 02.09 .2007$, K. Ravikumar \& R. Vijayasankar 107413, (FRLH).

Crotalaria assamica Benth., London J. Bot. 2: 481. 1843; Baker in Hook.f., Fl. Brit. Ind. 2: 75. 1876; Sanjappa, Leg. India 116. 1992; Ansari, Crotalaria Ind. 258. 2008. C. sericea Burm.f., Fl. Indica t. 48. f. 1. 1768. Type: t. 48 of protologue!. C. burmanni DC., Prodr. 2: 126. 1825. [Leguminosae; Plates 1 b \& III a]

Herbs, $1.5-2 \mathrm{~m}$ tall; stem terete, densely sericeous, pale yellow-brown. Leaves simple, oblong-oblonceolate, $4.5-7 \times 1.3-2.2 \mathrm{~cm}$, cuneate to subacute at base, entire at margins, obtuse to apiculate at apex, glabrous above, densely sericeous beneath; lateral nerves 8-9 pairs; petioles c. $2 \mathrm{~mm}$ long. Stipules absent. Bracts linear-lanceolate, $c .5 \mathrm{~mm}$ long, sericeous. Bracteoles linear, c. $4 \mathrm{~mm}$ long, glabrescent. Fruiting pedicles thick, up to $1.3 \mathrm{~cm}$ long. Calyx deeply 5-lobed, densely hairy outside, glabrous inside. Pods cylindrical, oblonceolate, c. 6 x 2 $\mathrm{cm}$ long, hooked at apex, glabrous, shiny, brown when dry; seeds reniform, c. 22, each c. $5 \mathrm{x}$ $4 \mathrm{~mm}$, hard, shiny, black.

Fruiting: December - February.

Habitat: Common in evergreen forest; growing with Chromolaena odorata (L.) R.M. King \& H. Rob., Coriaria nepalensis Wall., Erythrina stricta Roxb., Hedychium spicatum Sm., Rorippa montana (Wall. ex Hook. f. \& Thomson) Small and Rubus ellipticus Sm. 

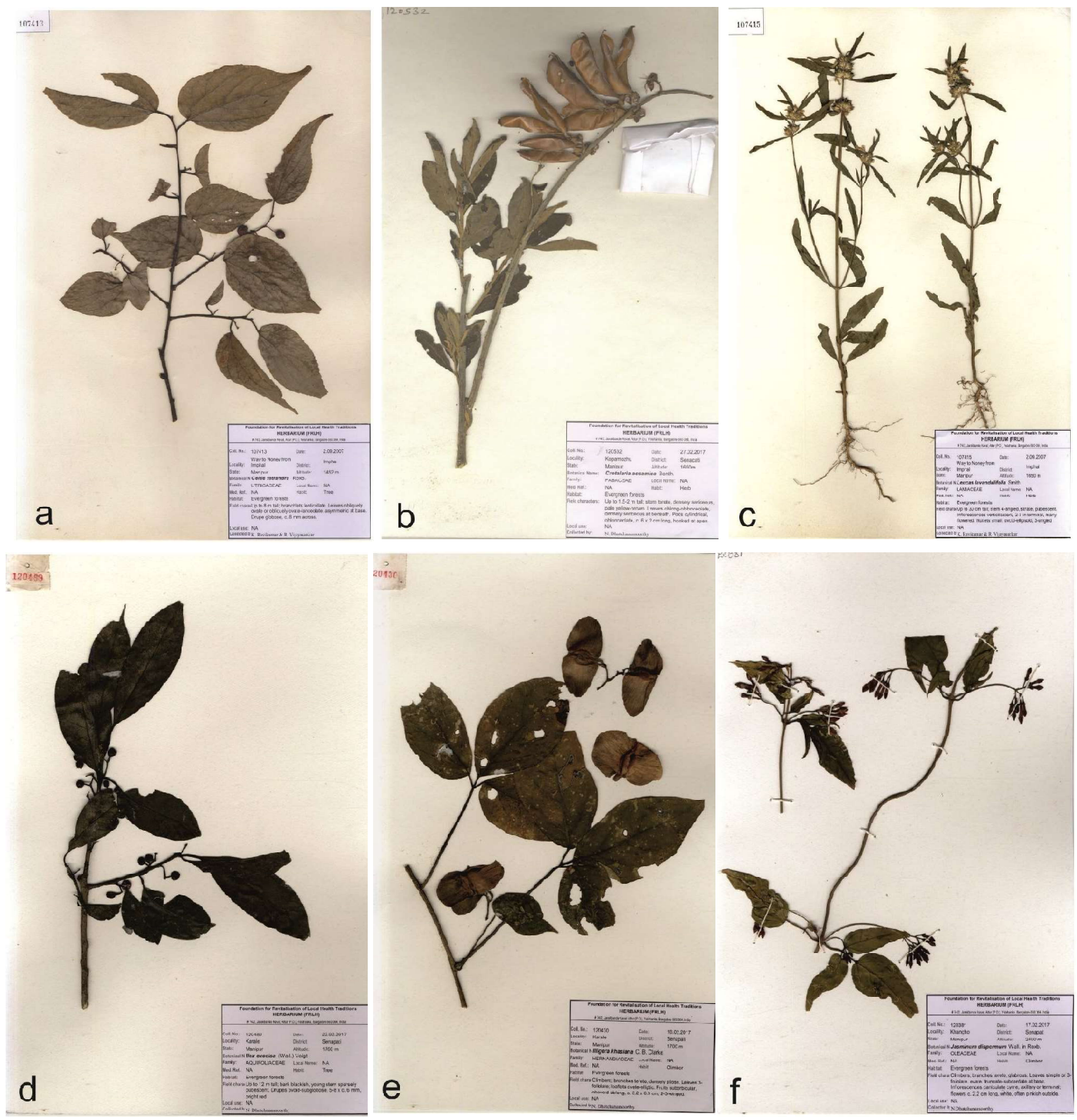

PLATE - I. Voucher specimens of newly recorded plants from Maniour: a. Celtis tetrandra; b. Crotalaria assamica; c. Leucas lavandulifolia; d. Ilex excelsa; e. Illigera khasiana; f. Jasminum dispermum.

Distribution: China, Laos, Myanmar, Malaysia, Philippines, Taiwan, Thailand, Vietnam and India. In India, it has been reported from Assam, Nagaland, Meghalaya and now from Manipur.

Specimens examined: India: Manipur, Senapati District, Kopamozhu, 1650 m, 24. 02. 2017, N. Dhatchanamoorthy 120532 (FRLH).

Leucas lavandulifolia Sm., Cycl. 20(2): 2. 1812; Mukerjee in Rec. Bot. Surv. India 14(1): 167. 1940; Singh, Ind. Leucas 104. 2001. Leonurus indicus L., Sp. Pl. ed. 2. 817. 1763. Phlomis linifolia Roth, Nov. Pl. Sp. 260. 1821. Leucas linifolia (Roth) Spreng., Syst. Veg. 2: 743. 1825; DC., Prodr. 12: 533. 1848; Hook.f., Fl. Brit. Ind. 4: 690. 1885. Phlomis zeylanica sensu Roxb., Fl. Ind. 3: 9. 1832, non L. 1753. P. ceilanica sensu Blanco, Fl. Filip. 475. 1837, non L. 1753 'zeylanica'. Hetrepta lavandulifolia (SM) Rafin., Fl. Tellur. 3: 88. 
1837. Leucas indica (L.) Vatke in Oesterr. Bot. Zeits. 25: 95. 1875, non R.Br. ex SM. 1812 based on Phlomis indica L. 1753. L. linifolia (Roth) Spreng. var. decipiens Hook.f., Fl. Brit. Ind. 4: 691. 1885. L. lavandulifolia SM. var. decipiens (Hook.f.) Chandr. \& Srin. in Bull. Bot. Surv. India 17: 165. 1978. [Lamiaceae; Plate I c]

Herbs, up to $40 \mathrm{~cm}$ tall; stems 4 -angled, striate, pubescent. Leaves elliptic or elliptic-oblong, linear-lanceolate, $30-55 \times 8-15 \mathrm{~mm}$, cuneate-acute at base, serrulate at margins, obtuse at apex; lateral nerves $c$. 4 pairs; petioles flat, $c$. $5 \mathrm{~mm}$ long. Inflorescences verticillasters, 2-3 in terminal, many flowered. Flower-head $c .10 \mathrm{~mm}$ across. Bracts linear-lanceolate, $c .1 \mathrm{x}$ $0.1 \mathrm{~mm}$, cuspitate at apex, pubescent, brownish. Calyx unequal, 8-lobed, 8-10-ribbed; tube cylindrical or campanulate, $c .8 \times 3 \mathrm{~mm}$ long; teeth lower ones short and upper one slightly long, acuminate, densely appressed pubescent. Corolla white, $c .10 \mathrm{~mm}$, outside silky hairy, glabrous inside. Nutlets small, ovoid-ellipsoid, 3-angled, c. $1 \mathrm{~mm}$, pale yellowish at base, purplish-black above, glabrous.

Flowering \& Fruiting: Throughout the year.

Habitat: Common in evergreen forest; growing with Brassaiopsis hainla (Buch.-Ham.) Seem., Chromolaena odorata (L.) R.M. King \& H. Rob. and Oxyspora paniculata (D. Don) DC.

Distribution: Africa, Bangladesh, Bhutan, China, Indonesia, Malesia, Mauritius, Myanmar, Philippines, Thailand and India. In India, it has been reported earlier from entire tropical regions, Western Himalaya, North-Eastern states except Manipur state.

Specimens examined: India: Manipur, Imphal District, way to Noney from Imphal, $1650 \mathrm{~m}$, 02.09.2017, K. Ravikumar \& R. Vijayasankar 107415 (FRLH).

Ilex excelsa (Wall.) Voigt, Hort. Suburb. Calcutt. 347. 1845; Hook.f., Fl. Brit. India. 1: 603. 1875. Cassine excels Wall., F1. India 2: 376. 1824. I. doniana DC., Prodr. 2: 644. 1824; Kanjilal et al., Fl. Ass. 1: 257. 1936. [Aquifoliaceae; Plates I d \& III b]

Trees, up to $12 \mathrm{~m}$ tall; bark blackish, young stems sparsely pubescent. Leaves ellipticoblong to lanceolate, $6-11 \times 2.5-3.5 \mathrm{~cm}$, cuneate-subacute at base, entire and margin recurved, acute-acuminate at apex; lateral nerves 8-13, glabrous above, sparsely pubescent along midrib beneath; petioles twisted, $1-1.5 \mathrm{~cm}$ long, minutely pubescent. Drupes ovoidsubglobose, 5-8 x c. $6 \mathrm{~mm}$, bright red when mature; pyrenes 3-5, ellipsoid, angled, c. 2 striate, glabrous.

Fruiting: January - March.

Habitat: Rare in evergreen forest; growing with Brassaiopsis hainla (Buch.-Ham.) Seem., Euodia fraxinifolia (D.Don) Hook.f., Terminalia bellirica (Gaertn.) Roxb. and Ziziphus glabrata B. Heyne ex Roth.

Distribution: Bhutan, China, Nepal, Pakistan and India. In India, it has been reported from Arunachal Pradesh, Assam, Himachal Pradesh, Jammu \& Kashmir, Meghalaya, Punjab, Sikkim, Uttar Pradesh and now from Manipur state.

Specimens examined: INDIA: Manipur, Senapati District, Karale, 1760 m, 22.02.2017, N. Dhatchanamoorthy 120488 (FRLH).

Illigera khasiana C.B.Clarke in Hook.f., Fl. Brit. India. 2: 461. 1879; Gamble, Man. Ind. Timp. 350. 1902; Brandis, Ind. Trees 542. 1906; Kanjillal et al., Fl. Assam 4: 103. 1940; Haridasan \& Rao, Fl. Meghalaya 2: 744. 1987. [Hernandiaceae; Plates I e \& III c] 


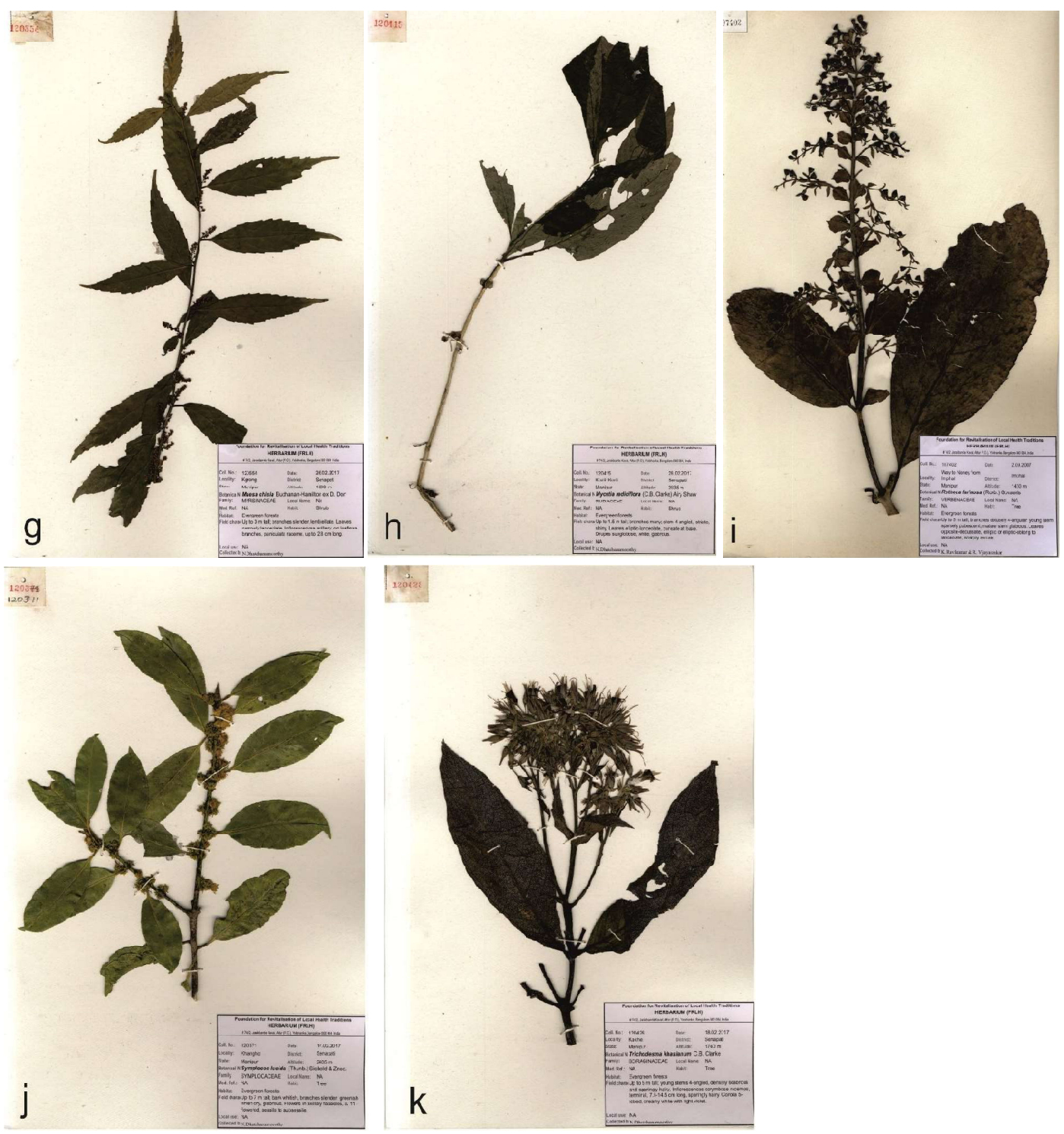

PLATE - II. Voucher specimens of newly recorded plants from Manipur: g. Maesa chisia; h. Mycetia radiciflora; i. Rotheca farinosa; j. Symplocos lucida; k. Trichodesma khasianum

Climbers; branches terete, densely pilose. Leaves 3-foliolate; central leaf ovate-elliptic, 8-13 $\mathrm{x}$ 4-6 cm, rounded, slightly oblique at base, entire at margins, acute-acuminate at apex, sparsely hairy along midrib on both sides; lateral nerves $c$. 5; lateral leaflets ovate, 7-8.5 x 3$4.5 \mathrm{~cm}$, oblique, slightly rounded at base, acute-acuminate at apex; lateral nerves $c .6$ pairs; petioles thick, striate, up to $11 \mathrm{~cm}$ long, glabrescent; petiolules 5-10 mm long. Fruits suborbicular, obovoid-oblong, c. 3 × $6 \mathrm{~cm}$, 2-3-winged, two wings broad, third one is short, glabrous.

Flowering: January - March.

Habitat: Rare in evergreen forests; growing with Capparis sp., Desmodium sequax Wall., Dumasia villosa DC., Meizotropis buteiformis Voigt, Oreocnide frutescens (Thunb.) Miq. and Tetrastigma obtectum (Wall. ex M.A. Lawson) Planch. ex Franch. 
Distribution: China, Malaysia, Myanmar and India. In India, it has been reported from Arunachal Pradesh, Assam, Meghalaya and now from Manipur.

Specimens examined: INDIA: Manipur, Senapati District, Kakhe, 1740 m, 18.02.2017, N. Dhatchanamoorthy 120430 (FRLH).

Jasminum dispermum Wall. in Roxb., Fl. Ind. 1: 99. 1820; C.B. Clarke in Hook.f., Fl. Brit. Ind. 3: 602. 1882; Kanjilal et al., Fl. Assam 3: 232. 1939; Haridasan \& Rao, Fl. Meghalaya 2: 586. 1987. [Oleaceae; Plates I f \& III d]

Climbers; branches terete, glabrous. Leaves simple or 3-foliolate; simple leaf: ovate, $c$. $4.7 \times 2.7 \mathrm{~cm}$, truncate-subcordate at base, margin entire, acute at apex, glabrous, 3-nerved at base; lateral nerves $c .5$; petioles $c .7 \mathrm{~mm}$ long, glabrescent; in compound leaf: lateral leaflets small, ovate, $3-5 \times 1.5-2.5 \mathrm{~cm}$, subcordate, truncate at base, acute, mucronate at apex, glabrous; 3-nerved at base; lateral nerves c. 5; petiole c. $4 \mathrm{~mm}$ long, glabrous or minutely hirsute, central leaf: broadly ovate, $6.5-9.7 \times 3.6-4.2 \mathrm{~cm}$, subcordate-truncate at base, margin entire, acute-acuminate, mucronate at apex; petioles twisted, thick, c. $2.4 \mathrm{~cm}$ long, minutely hirsute; petiolule $c .2 \mathrm{~cm}$ long, sparsely hirsute. Inflorescences paniculate cyme, axillary or terminal; peduncle pendulous, up to $7 \mathrm{~cm}$ long, minutely striate, glabrous. Flowers c. $2.2 \mathrm{~cm}$ long, white, often pinkish outside; bracts filiform-linear, 3-5 mm long, broadened at base, glabrous, black when dry; bracteoles linear-subulate, c. $2 \mathrm{~mm}$ long, slightly broadened at base, glabrous, acute at apex. Calyx 5-lobed, cup like, tube c. $3 \mathrm{~mm}$ across, glabrous, brown-blackish when dry, teeth slightly triangular at base, 1-2 mm long, sharply mucronate, $c .1 \mathrm{~mm}$ long, glabrous. Corolla salverform; tube, erect, $c .16 \times 2 \mathrm{~mm}$; lobe 5, obovate-oblong, glabrous. Stamens 2; filament flat, 1-2 mm long; anthers ovoidoblong, c. $2 \mathrm{~mm}$. Ovary ovoid, c. $1 \mathrm{~mm}$; style filiform, glabrous, c. $1 \mathrm{~cm}$; stigma 2-lobed, subequal, 1-2 mm long, glabrous. Berrys ovoid-globose, $c$. 7-10 x 5-6 mm, glabrous, purpleblackish when ripe; seeds 1-2, ovoid-suborbicular, $c .7$ x $5 \mathrm{~mm}$ long, brown-blackish, glabrous.

Flowering \& Fruiting: February - April.

Habitat: Rare in evergreen forests; growing with Ainsliaea latifolia (D.Don) Sch.Bip., Artemisia vulgaris L., Cyclea bicristata (Griff.) Diels, Phlogacanthus pubinervius T. Anderson and Senecio scandens Buch.-Ham. ex D.Don

Distribution: Bhutan, Kashmir, Nepal and India. In India, it has been reported form Arunachal Pradesh, Assam, Meghalaya and now from Manipur.

Specimens examined: INDIA: Manipur, Senapati District, Khangho, 2405 m, 17. 02. 2017, N. Dhatchanamoorthy 120381 (FRLH).

Maesa chisia Buch.-Ham. ex D.Don, Prodr. Fl. Nepal. 148. 1825; C.B. Clarke in Hook.f., Fl. Brit. Ind. 3: 509. 1882; Kanjilal et al., Fl. Assam 3: 1939; Giri et al., Fl. Arunachal Pradesh 2: 121. 2008. [Primulaceae; Plates II g \& III e]

Shrubs, up to $3 \mathrm{~m}$ tall; branches slender, lenticellate. Leaves narrowly lanceolate, 6.5 - 11.5 x $2-2.5 \mathrm{~cm}$, cuneate-acute at base, serrulate at margin, acuminate-caudate at apex, glabrous; lateral nerves $c$. 6 pairs; petioles thick, 5-10 mm long. Inflorescences axillary, on leafless branches, paniculate raceme, up to $2.8 \mathrm{~cm}$ long; pedicles thin, c. $2 \mathrm{~mm}$ long, grey pubescent. Bracts and bracteoles very similar, shortly ovate, c. $1 \mathrm{~mm}$ long, minutely grey pubescent. Calyx 5-lobed, minutely puberulous outside, glabrous inside; 2 outer sepals ovate, 1-3 mm, ciliate at margins; 3 inner sepals ovate, $1-2 \mathrm{~mm}$ long, ciliate at margin, acute at apex. Petals 5 equal; outer and inner similar, c. $1.2 \mathrm{~mm}, 3-5$-nerved at base, ciliate at margin, obtuse at apex. Stamens 5; filaments equal, c. $1 \mathrm{~mm}$, glabrous; anthers obovoid, c. $1 \mathrm{~mm}$, glabrous. 

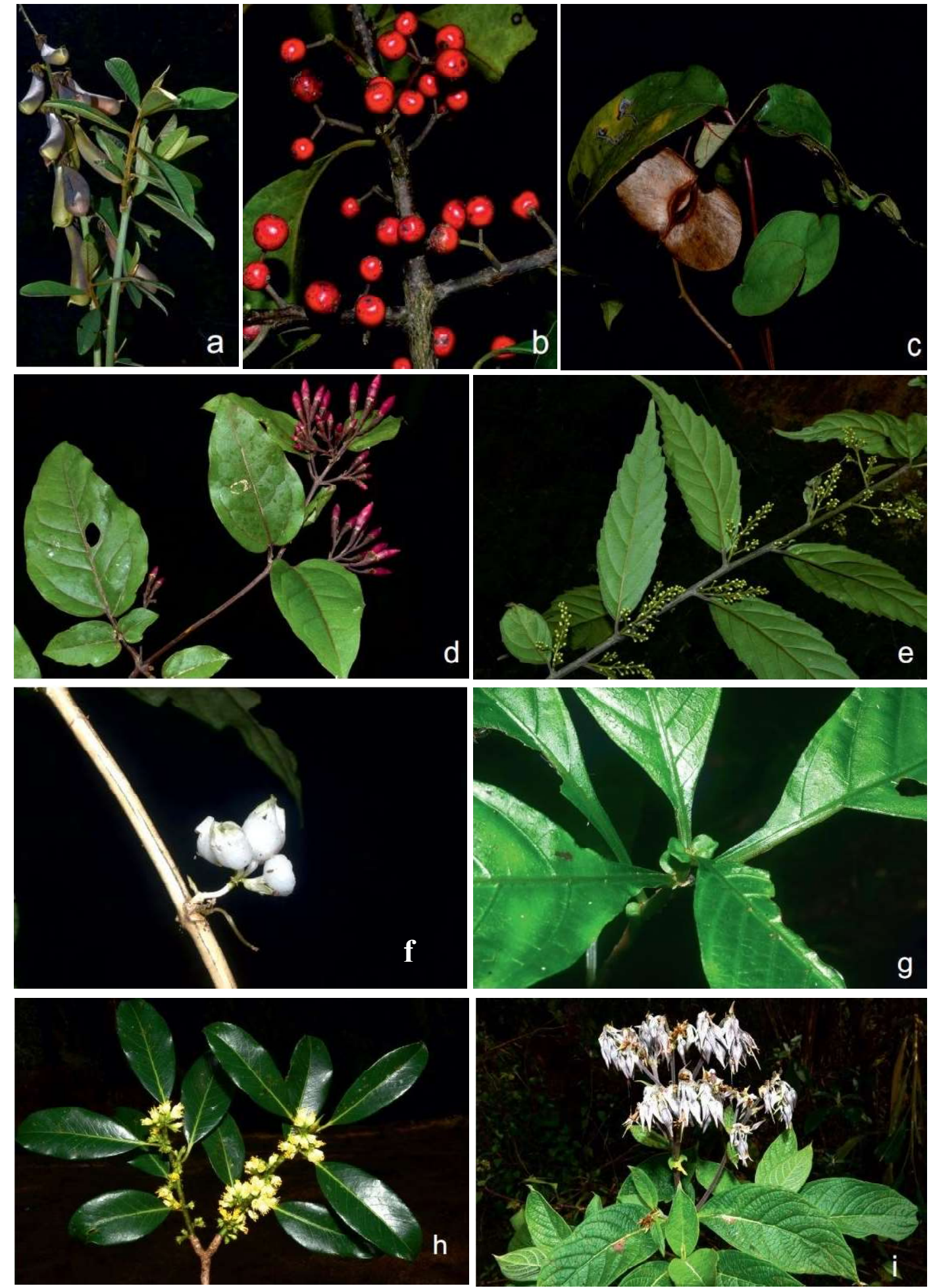

PLATE - III. Photographs of newly recorded plant from Manipur: a. Crotalaria assamica; b. Ilex excels; c. Illigera khasiana; d. Jasminum dispermum; e. Maesa chisia; f \& g. Mycetia radiciflora; h. Symplocos lucida; i. Trichodesma khasianum 
Ovary ovoid-obovoid, c. $2 \mathrm{~mm}$, glabrous, dark brown when dry; style flat, c. $1 \mathrm{~mm}$ long, glabrous; stigma truncate, glabrous, brownish.

Flowering: February - May.

Habitat: Less common in evergreen forest; growing with Clerodendrum wallichii Merr., Cynoglossum micranthum Poir., Flemingia macrophylla (Willd.) Merr., Osyris arborea Wall. ex A.DC. and Solanum erianthum D. Don

Distribution: Bhutan, Myanmar, Nepal and India. In India, it has been reported from Arunachal Pradesh, Assam, Nagaland and now from Manipur.

Specimens examined: INDIA: Manipur, Senapati District, Kgrong, 1830 m, 26.02.2017, N. Dhatchanamoorthy 120554 (FRLH).

Mycetia radiciflora (C.B. Clarke) Airy Shaw in Kew Bull. 19(3): 509. 1965; Deb in Bull. Bot. Surv. India 28(1-4): 124. 1988. Silavianthus radiciflora C.B. Clarke in J. Linn. Soc. Bot. 25: 32. 1889; Burkill in Rec. Bot. Surv. India 10: 51, 78, 79, 297. 1925; Chowdhery et al., Fl. Arunachal Pradesh 1: 591. 1996. [Rubiaceae; Plates II h \& III f, g]

Shrubs, up to $1.5 \mathrm{~m}$ tall; branches many; stems 4-angled, striate, shiny. Leaves ellipticlanceolate, $12-14.5 \times 3.5-5.5 \mathrm{~cm}$, cuneate at base, entire at margins, acute-acuminate at apex, upper side sparsely puberulous, glaucous beneath; lateral nerves $c .13$ pairs; petioles $c$. $6 \mathrm{~mm}$ long, glabrous. Stipules ovate-obovate, c. 7 x $5 \mathrm{~mm}$ long, subacute at apex, glabrous. Fruiting peduncle $5-15 \mathrm{~cm}$ long, twisted when dry. Drupes subglobose, $c .8 \times 5 \mathrm{~mm}$ across, white, glabrous.

Fruiting: January - March

Habitat: Rare in evergreen forest; growing with Coriaria nepalensis Wall., Drymaria cordata (L.) Willd. ex Schult., Eurya acuminata DC., Hedera nepalensis K. Koch, Lasianthus sikkimensis Hook.f., Urtica ardens Link and Youngia japonica (L.) DC.

Distribution: China, Myanmar and India. In India, it has been reported only from Arunachal Pradesh and now from Manipur state.

Specimens examined: INDIA: Manipur, Senapati district, Koziir Korii, 2036 m, 28.02.2017, N. Dhatchanamoorthy 120415 (FRLH).

Rotheca farinosa (Roxb.) Govaerts in World Checkl. Seed P1. 3(1): 15.1999. Clerodendrum farinosum (Roxb.) Wall. in [Numer. List 49, No. 1810. 1829, nom. nud.] ex Steudel, Nomencl. Bot. ed. 2, 1: 382. 1840; Daniel \& Rajendran in Taxon 42: 670. 1993 \& in Bull. Bot. Surv. India 35: 110, f. 1. 1997; Rajendran \& Daniel, Indian Verbenaceae, 102. 2002. Volkameria farinosa Roxburg, (Hort. Bengal. 46. 1814, nom. nud.), Fl. Ind. 3: 64. 1832. Clerodendrum venosum Wall. ex C.B. Clarke in Hook.f., Fl. Brit. India 4: 592. 1885, nom. illeg. incl. type of Volkameria farinosa Roxburgh (as 'Clerodendron'); Kanjilal et al., Fl. Assam 3: 490. 1939. [Lamiaceae; Plate II i]

Shrubs, up to $3 \mathrm{~m}$ tall; branches obtusely 4-angular, young stems sparsely pubescent, mature stems glabrous. Leaves opposite-decussate, 12.5-25 x 6.3-9.7 cm, elliptic or elliptic-oblong to lanceolate, sharply serrate, acuminate at apex, glabrous, membranous; lateral nerves 710; petioles $1.5-2 \mathrm{~cm}$ long. Fruiting inflorescences terminal, thyrses, up to $25 \mathrm{~cm}$ long, pubescent; pedicles flat, $c .3 \mathrm{~mm}$ long, with persistent bracts. Bracts ovate, $c .7 \times 2 \mathrm{~mm}$ long, minutely hairy. Fruiting calyx cup shaped, c. $4 \mathrm{~mm}$ across, glabrous. Drupes sub-globose, 2lobed, purplish-black. 
140 Additions to the flora of Manipur state

Fruiting: September - February.

Habitat: Common in Evergreen forest; growing with Artemisia vulgaris L., Litsea sp., Persicaria orientalis (L.) Spach, Rhododendron sp., Rhaphidospora sp. and Symplocos sp.

Distribution: Burma, Thailand and India. In India, it has been reported from Arunachal Pradesh, Assam, Nagaland, Meghalaya, Mizoram and now from Manipur state.

Specimens examined: INDIA: Manipur, Imphal District, way to Noney from Imphal, 1450 m, 02.09.2007, K. Ravikumar \& R. Vijayasankar 107402 (FRLH).

Symplocos lucida (Thunb.) Siebold \& Zucc., Fl. Jap. 1: 55, t. 24. 1835, p.p. excl. syn. Myrtus laevis Thunb.; Noot. in Steenis, Fl. Males. 8(2): 264. 1977. Laurus lucida Thunb., Fl. Jap. 174. 1784. Symplocos theaefolia D. Don, Prodr. Fl. Nep. 145. 1825; C. B. Clarke in Hook. f., Fl. Brit. Ind. 3: 575. 1882; Kanjilal et al., Fl. Assam 3: 218. 1939. [Symplocaceae; Plates II j \& III h]

Trees, up to $7 \mathrm{~m}$ tall; bark whitish, branches slender, greenish when dry, glabrous. Leaves elliptic-lanceolate, 6-9.5 x 2.5-3.5 cm, cuneate, retuse-rounded at base, shortly serrate at margins, acute-acuminate to mucronate at apex; lateral nerves $c$. 11 pairs; petioles twisted, up to $1.6 \mathrm{~cm}$ long, glabrous. Flowers in axillary fascicles, $c .11$-flowered, sessile to subsessile. Calyx 5-lobed; lobes obovoid, c. $1 \mathrm{~mm}$ long, obtuse at apex, glabrous. Petals 5, pale white, obovoid-suborbicular, acute at base, $c .5 \times 3 \mathrm{~mm}$, glabrous. Stamens c. 20; filaments unequal, flat, filiform, c. $5 \mathrm{~mm}$ long, glabrous; anthers ovoid, c. $0.1 \mathrm{~mm}$, yellowish. Ovary obovoid, $c$. $1 \mathrm{~mm}$, glabrous; styles slender, c. $5 \mathrm{~mm}$ long, glabrous; stigma subcapitate, black when dry, glabrous.

Flowering: January - February.

Habitat: Common in Evergreen forest; growing with Agapetes borii Airy Shaw, Ardisia crenata Sims, Cleisostoma filiforme (Lindl.) Garay, Piper hymenophyllum (Miq.) Wight and Schefflera hypoleuca (Kurz) Harms.

Distribution: Bhutan, China, Cambodia, Indonesia, Japan, Laos, Malaysia, Myanmar, Thailand, Vietnam and India; In India, it has been reported from Arunachal Pradesh, Assam, Meghalaya and now from Manipur state.

Specimens examined: INDIA: Manipur, Senapati District, Khangho, 2405 m, 17.02.2017, N. Dhatchanamoorthy 120371 (FRLH).

Trichodesma khasianum C.B. Clarke in Hook.f., Brit. Ind 4: 154. 1883; Kanjilal et al., Fl. Assam 3: 338. 1939; R.N. Banerjee \& Pramanik in Bull. Bot. Surv. India 17(1-4): 118. 1978; A.S. Chauhan in Hajra, Contrib. Fl. Namdapha 226. 1996. [Boraginaceae; Plates II k \& III i]

Small trees, up to $5 \mathrm{~m}$ tall; young stems 4 -angled, densely scabrous and sparingly hairy. Leaves elliptic-lanceolate, 7-20 x 2-7 cm, cuneate at base, entire at margins, acuminate at apex, densely scabrous and tuberculate above, sparsely tubercled beneath; lateral nerves 811; petioles thick, $c .1 \mathrm{~cm}$ long, broaden at base, hispid hairy. Inflorescences corymbose racemes, terminal, $7.5-14.5 \mathrm{~cm}$ long, sparingly hairy. Flowers erect or pendulous; primary peduncles, up to $5 \mathrm{~cm}$ long; secondary peduncles, up to $3 \mathrm{~cm}$ long; pedicels thin, $1-2 \mathrm{~cm}$ long, pubescent pale brownish. Calyx 5-lobed, tube shorter then corolla tube, c. $5 \mathrm{~mm}$, lobe lanceolate, $c .18 \times 2 \mathrm{~mm}$, acuminate at apex, rusty pubescent outer and inside. Corolla 5lobed, creamy white with light violet; lobes linear-lanceolate, $c .19 \times 5 \mathrm{~mm}$ long, acuminate at 
apex, glabrous. Stamens 5; anther flat, elongate, connectives inserted from corolla, c. $9 \mathrm{~mm}$ long, patently white hairy. Ovary 4-celled, c. $3 \mathrm{~mm}$ long, c. $3.2 \mathrm{~mm}$ long, sparsely hairy.

Flowering: February-May.

Habitat: Rare in evergreen forest; growing with Balanophora fungosa J.R.Forst. \& G.Forst., Clerodendrum wallichii Merr., Elaeagnus conferta Roxb., Maesa montana A. DC., Rubia sikkimensis Kurz, Sarcopyramis napalensis Wall., Sarcococca coriacea Sweet and Stephania elegans Hook.f. \& Thomson

Distribution: Myanmar, China, Vietnam and India. In India, it has been reported from Arunachal Pradesh, Assam and now from Manipur.

Specimens examined: INDIA: Manipur, Senapati District, Kakhe, 1740 m, 18.02.2017, N. Dhatchanamoorthy 120428 (FRLH).

\section{Acknowledgements}

The authors are thankful to Shri. Darshan Shankar, Vice Chancellor, FRLHT-TDU; Shri. D.K. Ved, IFS (Retd.) Advisor, FRLHT-TDU, Bangalore for providing facilities, constant support and encouragements; the Ministry of Environment and Forests \& Climate Change, New Delhi for financial support under Centre of Excellence project. Special thanks to Mr. Athikho Kreni, Mr. Nepuni Lokho, Mr. Adani Dikho and Mr. A. Ashiili for their kind help in field survey and specimen processing. Thanks are also due to Ms. Suganthi Fathima, Research Associate, FRLHT-TDU for meticulously setting the plates.

\section{LITERATURE CITED}

Ansari, A.A. 2008. Crotalaria L. in India. Bishen Singh Mahendra Pal Singh, Dehra Dun.

Balachandran, N. \& Ravikumar, K. 2014. Additions to the Flora of Manipur in North East of India. Pleione 8(1): 199 - 206.

Balakrishnan, N.P. 1981. Flora of Jowai and Vicinity Meghalaya. Vol. 1. Botanical Survey of India, Botanic Garden Howrah, Calcutta, India.

Balakrishnan, N.P. 1983. Flora of Jowai and Vicinity Meghalaya. Vol 2. Botanical Survey of India, Botanic Garden Howrah, Calcutta, India.

Balakrishnan, N.P.; Chakrabarty, T.; Sanjappa, M.; Lakshminarasimhan, P. \& Singh, P. (eds.) 2012. Flora of India, Loranthaceae-Daphniphyllaceae. Vol. 23. Botanical Survey of India, Kolkata.

Chauhan, A.S. 1996. Contribution to the Flora of Namdapha, Arunachal Pradesh. Botanical Survey of India, Calcutta.

Chaudhuri, A.B. 1993. Forest Plants of Eastern India. Ashish Publishing House, New Delhi.

Chowdhery, H.J. \& Wadhwa, B.M. 1984. Flora of Himachal Pradesh. Vol. 2. Botanical Survey of India, Department of Environment, New Delhi.

Chowdhery, H.J.; Giri, G.S.; Pal, G.D.; Pramanik, A. \& Das, S.K. 1996. Materials for the Flora of Arunachal Pradesh. Vol. 1. Botanical Survey of India, Kolkata.

Chowdhery, H.J.; Giri, G.S.; Pal, G.D.; Pramanik, A. \& Das, S.K. 2008. Materials for the Flora of Arunachal Pradesh. Vol. 2. Botanical Survey of India, Kolkata.

Deb, D.B. 1981. Flora of Tripura State. Vol. 1. Ophioglossaceae-Staphyleaceae. Today \& Tomorrow's Printers and Publishers. 
Deb, D.B. 1983. The Flora of Tripura State. Vol. 2. Buddlejaceae-Gramineaceae (Poaceae). Today \& Tomorrow's Printers and Publishers.

Devi, S. Th.; P.D. Singh, B.G.; Somkuwar, Thorat \& Kumar, S.S. 2018. Oberonia pachyrhachis (Orchidaceae): a new addition to the flora of Manipur, India. Jardin Botanique de Guyane, Richardiana Vol. 2: 1 - 7 .

Hajra, P.K.; Rao, R.R.; Singh, D.K. \& Uniyal, B.P. 1995a. Flora of India. AnthemideaeHeliantheae. Vol. 12. Botanical Survey of India, Calcutta.

Hajra, P.K.; Rao, R.R.; Singh, D.K. \& Uniyal, B.P. 1995b. Flora of India. InuleaeVernonieae. Vol. 13. Botanical Survey of India, Calcutta.

Haridasan, K. \& Rao, R.R. 1985. Forest Flora of Meghalaya. Vol. 1. Bishen Singh Mahendra Pal Singh, Dehra Dun.

Haridasan, K. \& Rao, R.R. 1987. Forest Flora of Meghalaya. Vol. 2. Bishen Singh Mahendra Pal Singh, Dehra Dun.

Hooker, J.D. 1872-1897. The Flora of British India. Vols. 1-7. L. Reeve \& Co., Kent, London.

Kanjilal, U.N., Kanjilal, P.C., Das, A., De, R.N. \& Bor, N.L. 1934-1940. Flora of Assam. Vols. 1-5. Assam Government Press, Shillong.

Kazhuhrii Eshuo, C. \& S.K. Chaturvedi. 2011. Ceropegia longifolia Wallich (Apocynaceae: Asclepiadoideae) - A new record for Manipur, India. Pleione 5(1): 201 - 204.

Kumar, S. \& Singh, V. 2001. Asteraceae of Sikkim, Deep Publications, New Delhi.

Moaakum \& Dey, S. 2013. Rediscovery of Hedychium longipedunculatum A.R.K. Sastry \& D.M. Verma (Zingiberaceae) from Nagaland, India. Pleione 7(1): 290 - 294.

Moaakum, Dey, S.; Chaturvedi, S.K. \& Jamir, N.S. 2014. Diversity of the genus Hoya R.Brown (Apocynaceae: Asclepediaceae) in Tuensang and Zunheboto district of Nagaland, India. Pleione 8(2): 391 - 395.

Naithani, H.B. 1990. Flowering Plants of India, Nepal \& Bhutan. A Surya Publication.

Oleg Polunin \& Adam Stainton. 1997. Flowers of the Himalaya, Oxford University Press, Oxford.

Sanjappa, M. 1992. Legumes of India. Bishen Singh Mahendra Pal Singh, Dehra Dun.

Sharma, B.D. \& Sanjappa, M. 1993. Flora of India. Portulacaceae-Ixonanthaceae. Vol. 3. Botanical Survey of India, Calcutta.

Singh, N.P.; Vohra, J.N.; Hajra, P.K. \& Singh, D.K. 2000a. Flora of India. OlacaceaeConnaraceae. Vol. 5. Botanical Survey of India, Calcutta.

Singh, N.P.; Chauhan, A.S. \& Mondal, M.S. 2000b. Flora of Manipur. Vol. 1. Botanical Survey of India, Kolkata.

Singh, V. 2001. Monograph on Indian Leucas R.Br. (Dronapushpi) Lamiaceae. Scientific Publishers (India), Jodhpur.

Singh, N.P.; Singh, K.P. \& Singh, D.K. 2002. Flora of Mizoram. Vol. 1. Botanical Survey of India, Kolkata, Ministry of Environment and Forest. New Delhi.

Thongam, B.; Sarangthem, N. \& Konsam, B. 2013a. Zingiber kerrii (Zingiberaceae): A New Record for India from Manipur. Taiwania, 58(4): 291294.

Thongam, B.; Konsam, B. Bipin \& Sarangthem, N. 2013b. Globba sherwoodiana (Zingiberaceae) - A new record for India from Manipur. Rheedea, 23(1): 34 - 36. 